\title{
Effectiveness of botulinum toxin injection in the treatment of de novo OAB symptoms following midurethral sling surgery
}

\author{
Pawel Miotla $^{1} \cdot$ Konrad Futyma $^{1} \cdot$ Rufus Cartwright $^{2} \cdot$ Michal Bogusiewicz $^{1} \cdot$ \\ Katarzyna Skorupska $^{1}$ • Ewa Markut-Miotla ${ }^{3}$. Tomasz Rechberger ${ }^{1}$
}

Received: 14 July 2015 / Accepted: 24 August 2015 / Published online: 12 September 2015

(C) The Author(s) 2015. This article is published with open access at Springerlink.com

\begin{abstract}
Introduction and hypothesis Intravesical onabotulinumtoxinA $\left(\right.$ Botox $\left.^{\mathbb{R}}\right)$ is effective for idiopathic overactive bladder $(\mathrm{OAB})$ symptoms. Our primary objective was to compare the efficacy of onabotulinumtoxinA for women with de novo OAB after midurethral sling (MUS) surgery and women with idiopathic OAB.

Methods Women enrolled in this prospective study had idiopathic $(n=53)$ or de novo $(n=49)$ OAB symptoms after MUS, with at least one episode of urgency urine incontinence per day. OnabotulinumtoxinA (100 U) was administered in 20 intradetrusor injections. Postvoid residual volumes were checked at 2, 4 and 12 weeks. Participants completed a 3day bladder diary and the King's Health Questionnaire (KHQ) before and 12 weeks after treatment.

Results After 12 weeks, 22 patients (41.5\%) in the idiopathic $\mathrm{OAB}$ and 19 patients $(38.8 \%)$ in the de novo OAB groups were completely dry. OnabotulinumtoxinA injections had a significant benefit within both groups $(p<0.001)$ to decrease both the daily numbers of voids $(-2.39$ and -2.0$)$ and incontinence episodes $(-1.38$ and -1.44$)$, with no significant
\end{abstract}

An abstract (submission: 2015-A-682-IUGA) with preliminary results of this study was accepted for poster presentation orally (OP 059) at the 2015 IUGA Meeting in Nice, France.

Pawel Miotla

pmiotla@wp.pl

1 2nd Department of Gynaecology, Medical University of Lublin, ul. Jaczewskiego 8, 20-954 Lublin, Poland

2 Department of Epidemiology and Biostatistics; Department of Urogynaecology, Imperial College London, London, UK

3 Department of Paediatric Nursing, Medical University of Lublin, Lublin, Poland difference between groups. We observed an increase of mean voided volume of $>90 \mathrm{ml}$ in both groups. Urinary retention was observed in four patients.

Conclusions We observed similar improvement in OAB symptoms after intravesical onabotulinumtoxinA injections within both groups. The rates of retention and requirement for catheterization even for women with a prior MUS were acceptable. These observational data provide evidence that onabotulinumtoxinA can effectively treat patients with $\mathrm{OAB}$ following stress urinary incontinence surgery.

Keywords Botulinum toxin - De novo OAB $\cdot$ Midurethral sling · Overactive bladder · Quality of life · Urgency incontinence

\section{Introduction}

Midurethral slings (MUS) are widely used as first-line surgical therapy for women with stress urinary incontinence (SUI). Rates of surgery have sharply increased over the last decade [1], with a recent estimate for the lifetime risk of SUI surgery of $13.6 \%$ [2]. With current demographic trends, it is expected that the number of MUS surgeries performed will continue to increase.

Overall, these minimally invasive procedures have a low incidence of complications. It was described that similarly to earlier procedures for SUI-e.g., Burch colposuspensioninsertion of an MUS may also be associated with the development of urge urinary incontinence (UUI) and storagerelated lower urinary tract symptoms (LUTS). Reports indicate that $6-8 \%$ of women treated with MUS will develop de novo overactive bladder (OAB) symptoms [3, 4].

New-onset storage LUTS significantly impact on subjective cure rates and patients' satisfaction with these procedures 
[5]. The mechanisms leading to symptoms of de novo OAB are still not well understood but may occur even after a first MUS surgery with correct tape placement [6]. In some cases, $\mathrm{OAB}$ symptoms may be attributed to excessive tension applied to the tape or its placement too close to the bladder neck [7-9]. After tape malposition, patients are likely to benefit from tape incision; however, most women will require pharmacological treatment. There are limited data regarding the efficacy of therapies, specifically in patients with de novo OAB after MUS.

Symptoms of idiopathic overactive bladder (OAB) affect $\sim 17 \%$ of women, and its prevalence increases with patient age, achieving $30.9 \%$ in elderly patients [10]. One of the most bothersome symptoms of OAB is UUI, followed by urgency and frequency [11]. The standard pharmacological treatment for idiopathic OAB starts with anticholinergic drugs or mirabegron intake $[12,13]$. Many patients with idiopathic $\mathrm{OAB}$ cannot be adequately managed with orally administered drugs. Recent evidence also suggests that antimuscarinic therapy provides less subjective benefit for patients with de novo compared with idiopathic OAB [14].

Intravesical injections of onabotulinumtoxinA or sacral nerve stimulation are widely used third-line options for $\mathrm{OAB}$. The efficacy of onabotulinumtoxinA (100 U) in the treatment of idiopathic $\mathrm{OAB}$ has been repeatedly shown in randomized clinical trials (RCTs) $[15,16]$. Compared with placebo, it significantly decreases daily micturition episodes, and the number of UUI episodes, and importantly also significantly improves health-related quality of life in patients with idiopathic OAB [15-18]. However, no studies have tested the benefits of onabotulinumtoxinA specifically for patients with de novo OAB after MUS procedures. Hence, we designed a study aiming to compare the efficacy and safety of intravesical onabotulinumtoxinA injections in women with de novo and idiopathic $\mathrm{OAB}$. The primary endpoints were changes in daily number of micturitions and UUI episodes from baseline to week 12. Secondary endpoints were changes in voided volume, differences in pad use, and changes in King's Health Questionnaire (KHQ) scores compared with baseline. Safety assessments included all common potential adverse events of botulinum toxin, including urinary retention, increased postvoid residual (PVR) volumes, and urinary tract infection (UTI).

\section{Materials and methods}

The study protocol was approved by our Institutional Board Review for the Medical University of Lublin, Poland. All participating patients were counselled regarding potential adverse effects of onabotulinumtoxinA; they gave written informed consent. Inclusion criteria were as follows: nonpregnant women $>18$ years of age; idiopathic $\mathrm{OAB}$ wet symptoms or de novo $\mathrm{OAB}$ wet symptoms (at least one urine leakage per day and more than eight micturition daily); lack of efficacy (at least 3 months) or intolerance to antimuscarinic therapy; stage 0 or 1 on the Pelvic Organ Prolapse Quantification (POP-Q) scale.

Exclusion criteria were mixed urinary incontinence (MUI) in the past; $\mathrm{OAB}$ symptoms before sling surgery; bladderoutlet obstruction $(\mathrm{BOO})$ after surgery [maximum flow $\left(\mathrm{Q}_{\max }\right)$ on uroflowmetry $<15 \mathrm{ml} / \mathrm{s}$ ]; UTI; neurologic disorders affecting bladder function; PVR volume $>100 \mathrm{ml}$; contraindications to onabotulinumtoxinA use; allergy to lidocaine; uncontrolled systemic diseases; contraindications to clean intermittent selfcatheterization; previous prolapse or anti-incontinence surgery (except midurethral sling surgery with transobturator approach; only one procedure in the past and at least 6 months before onabotulinumtoxinA treatment); previous onabotulinumtoxinA injections due to urological conditions. All participants were of European descent. From November 2011 to June 2014, 53 patients with idiopathic and 49 with de novo OAB after MUS surgery were enrolled. All women in the MUS group had been treated with transobturator tapes (Tsling, Polhernia) and were enrolled in our study a minimum of 6 months after that surgery. At enrollment, all patients had been treated for at least 3 months with anticholinergics - either without sufficient improvement in $\mathrm{OAB}$ symptoms, or with treatment limited by adverse events.

Participants completed 3-day bladder diaries, including a count of pads used. They also completed a validated translation of the KHQ for evaluation of incontinence-specific quality of life $(\mathrm{QoL})$ impairment, and they finally underwent a systematic clinical examination. Postvoid residual measurements using abdominal ultrasound (US) were performed in all patients before treatment. Urodynamic testing was assessed to confirm $\mathrm{OAB}$ symptoms and exclude the possibility of $\mathrm{BOO}$ and therefore the need for sling incision. Additionally for the sling group, transvaginal US was performed as described previously in order to exclude patients with tape malposition who could benefit from sling incision $[8,9]$. Two blinded evaluators reviewed outcome data before analyses.

Participants started with antibiotic prophylaxis (ciprofloxacin $500 \mathrm{mg}$ per os twice daily) for 1 day before the injection procedure and continued for 5 days after treatment. Thirty minutes before the procedure, all patients received a $100-\mathrm{ml}$ instillation of $2 \%$ lidocaine intravesically through a Foley catheter. Saline cystoscopy was performed using a 19-F rigid cystoscope (Karl-Storz). OnabotulinumtoxinA (100 U) in $10 \mathrm{ml} 0.9 \%$ sodium chloride was administered in 20 injections during cystoscopy, sparing the trigone, using a 25 -gauge injeTAK 198 DIS needle (Laborie). The tip of needle was inserted $3 \mathrm{~mm}$ into the bladder wall. Patients were discharged 2-3 h after injections and following a successful demonstration of voiding. PVR volumes were checked after 2 weeks, 1 month, and 3 months. Patients with elevated residual volumes 
(PVR $>100 \mathrm{ml}$ ) received extended antibiotic prophylaxis (e.g., nitrofurantoin $100 \mathrm{mg}$ three times per day). Patients completed a further bladder diary and the KHQ after 3 months.

Statistical analysis was performed with Statistica Statsoft, version 10 package, using the unpaired or paired Student $t$ test, the Mann-Whitney $U$ test, and the $\chi 2$ test, as appropriate. A $p$ value $<0.05$ was considered statistically significant throughout. The sample size was pragmatic, but post hoc power calculations based on the observed success rate, taking into account the decrease in mean number of incontinence episodes and frequency of micturitions as primary endpoints and using the $t$ test for paired samples, showed $>95 \%$ power at a two-sided significance level of 0.05 for each group.

\section{Results}

\section{Patient demographics}

Baseline demographic characteristics were similar between groups, with the exception of higher parity among women with a prior MUS (Table 1). One hundred and two patients completed treatment and were available with 12 weeks of follow-up (Fig. 1).

\section{Clinical effectiveness}

After 12 weeks, 22 (41.5\%) patients with idiopathic and $19(38.8 \%)$ with de novo OAB were completely dry on their 3-day bladder diaries, and the rates of dryness did not differ between groups $(p>0.05)$. Taking into account that all these patients had at least one episode of urine leakage per day at baseline, these data support high effectiveness of the procedure. OnabotulinumtoxinA injections significantly decreased the mean number of daily micturitions, incontinence episodes, and number of pads used, as well as increasing mean voided volume per micturition in both groups. Across all outcomes, efficacy was similar for patients with idiopathic and de novo OAB, with no statistically significant differences between groups (Table 2; Figs. 2 and 3).

Table 1 Demographic characteristics of patient groups

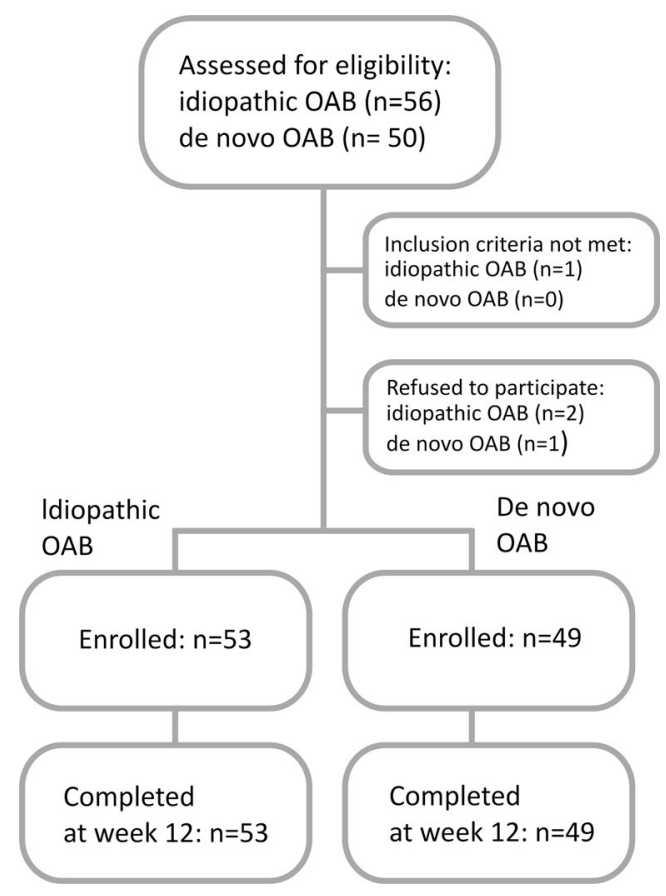

Fig. 1 Study participants

\section{Quality of life}

At the 12-week follow-up, participants in both groups reported lower values in all questionnaire domains compared with baseline (high KHQ scores represent greater QoL impairment). There were statistically significantly improvements at 3 months in each domain in both groups ( $p<0.001)$, including the General Health Perception domain. We found no statistically significant differences in QoL improvement between groups either for individual domains or for total KHQ score (Fig. 4).

\section{Safety}

The most frequently observed adverse event in both groups was the increase in residual volume, which was usually asymptomatic and clinically nonsignificant. Elevated residual volumes were most prevalent during the US examination performed 2 weeks after treatment. The number of patients with elevated PVR volumes decreased from week 2 to week 12 in both groups. The most severe adverse event was urinary retention, which was observed in four patients (one in the

\begin{tabular}{llll}
\hline Variable & Idiopathic OAB $(n=53)$ & De novo OAB after MUS $(n=49)$ & $p$ value \\
\hline Age $($ years $)$ & $58.7 \pm 15.3$ & $63.6 \pm 10.2$ & 0.06 \\
BMI $\left(\mathrm{kg} / \mathrm{m}^{2}\right)$ & $29.1 \pm 5.3$ & $30.1 \pm 5.0$ & 0.12 \\
Parity & $1.01 \pm 1.53$ & $1.75 \pm 2.53$ & $<0.001$ \\
Previous hysterectomy & $11(20.75)$ & $7(14.3)$ & 0.39 \\
\hline
\end{tabular}

Continuous variables are mean \pm standard deviation; categorical variables are $n(\%)$

$B M I$ body mass index, $O A B$ overactive bladder, $M U S$ midurethral sling 
Table 2 Pre- and posttreatment clinical parameters in patients with idiopathic and de novo overactive bladder (OAB) who received intravesical onabotulinumtoxinA injections

\begin{tabular}{lcc}
\hline Parameters & Idiopathic OAB $(n=53)$ & De novo OAB after MUS $(n=49)$ \\
\hline Number of micturitions/24 h (pretreatment) & $10.89 \pm 1.43$ & $10.94 \pm 1.34$ \\
Number of micturitions/24 h (posttreatment) & $8.50 \pm 2.05$ & $8.93 \pm 1.84$ \\
Number of UUI/24 h (pretreatment) & $1.87 \pm 0.86$ & $2.12 \pm 0.86$ \\
Number of UUI/24 h (posttreatment) & $0.49 \pm 0.65$ & $0.68 \pm 0.94$ \\
Volume voided (ml)/micturition (pretreatment) & $202.3 \pm 30.0$ & $199.0 \pm 35.7$ \\
Volume voided (ml)/micturition (posttreatment) & $295.7 \pm 62.1$ & $290.6 \pm 77.9$ \\
Number of incontinence pads used/24 h (pretreatment) & $3.07 \pm 0.97$ & $3.47 \pm 1.32$ \\
Number of incontinence pads used/24 h (posttreatment) & $1.37 \pm 0.88$ & $1.63 \pm 1.25$
\end{tabular}

All data presented as mean \pm standard deviation. Differences between pre- and posttreatment values within each group were statistically significant (all $p<0.001$ ). There were no statistically significant differences between groups

idiopathic group with a postvoid volume of $300 \mathrm{ml}$, and three in the de novo $\mathrm{OAB}$, two of whom had PVR $>350 \mathrm{ml}$ and one $310 \mathrm{ml}$ ); two cases in the de novo group required selfcatheterization for 1 month. In the other two patients, PVR decreased to $250 \mathrm{ml}$ and $280 \mathrm{ml}$ after one week, respectively. Urinary tract infections were rarely observed, at less than $4 \%$ in both groups. We did not observe any major complications, requiring readmission or reoperation, in either group (Table 3).

\section{Discussion}

We believe this study is the first to evaluate the effectiveness of onabotulinumtoxinA specifically for patients with de novo

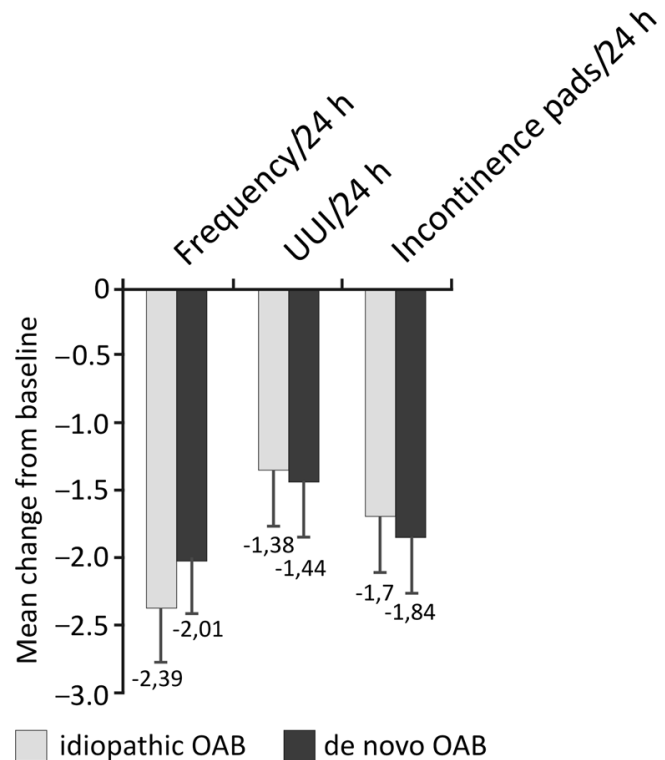

Fig. 2 Change from baseline in number of daily micturitions, urge incontinence episodes, and pads used in patients with idiopathic and de novo overactive bladder $(\mathrm{OAB})$ at week 12 after onabotulinumtoxin $\mathrm{A}$ injections. There were no statistically significant differences between idiopathic vs de novo OAB groups
OAB symptoms after MUS surgery. Our results demonstrate that the efficacy of onabotulinumtoxinA injections is comparable with efficacy for idiopathic $\mathrm{OAB}$, providing tape malposition is excluded. The primary endpoint of complete dryness measured at 12 weeks, observed in almost $39 \%$ of de novo $\mathrm{OAB}$ patients, demonstrated a high cure rate for UUI, with clinically relevant and statistically significant improvements in all secondary outcomes.

Women in the de novo group reported reductions in both the daily number of UUI episodes and the frequency of voiding, with an increase in voided volume $>90 \mathrm{ml}$. These objective findings are similar to data from recent randomized trials of onabotulinumtoxinA (100 U) injections in patients with idiopathic $\mathrm{OAB}[15,18]$. We also observed improvement

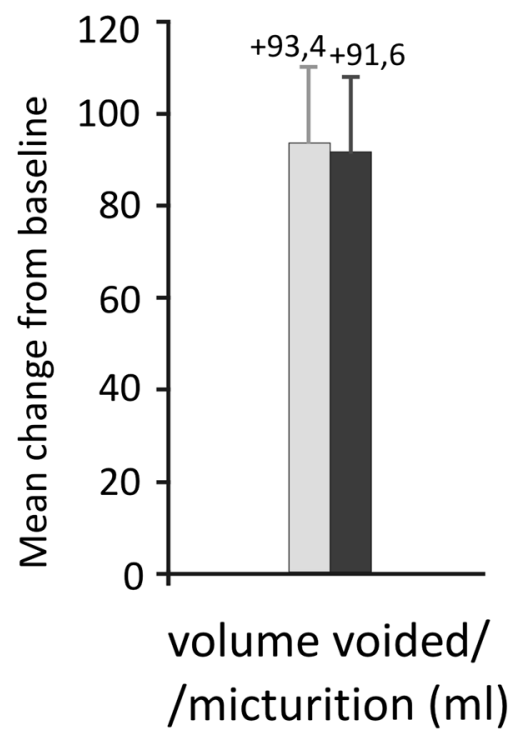

\section{idiopathic $O A B$}

\section{de novo OAB}

Fig. 3 Change from baseline in voided volume in patients with idiopathic and de novo overactive bladder $(\mathrm{OAB})$ at week 12 after onabotulinumtoxinA injections. There was no statistically significant difference between idiopathic and de novo OAB groups 
Fig. 4 Change from baseline in quality of life in the King's Health Questionnaire at week 12 after onabotulinumtoxinA injections. Differences between pre- and posttreatment values in each investigated group were highly statistically significant (all $p<0.001$ ). There were no statistically significant differences between groups

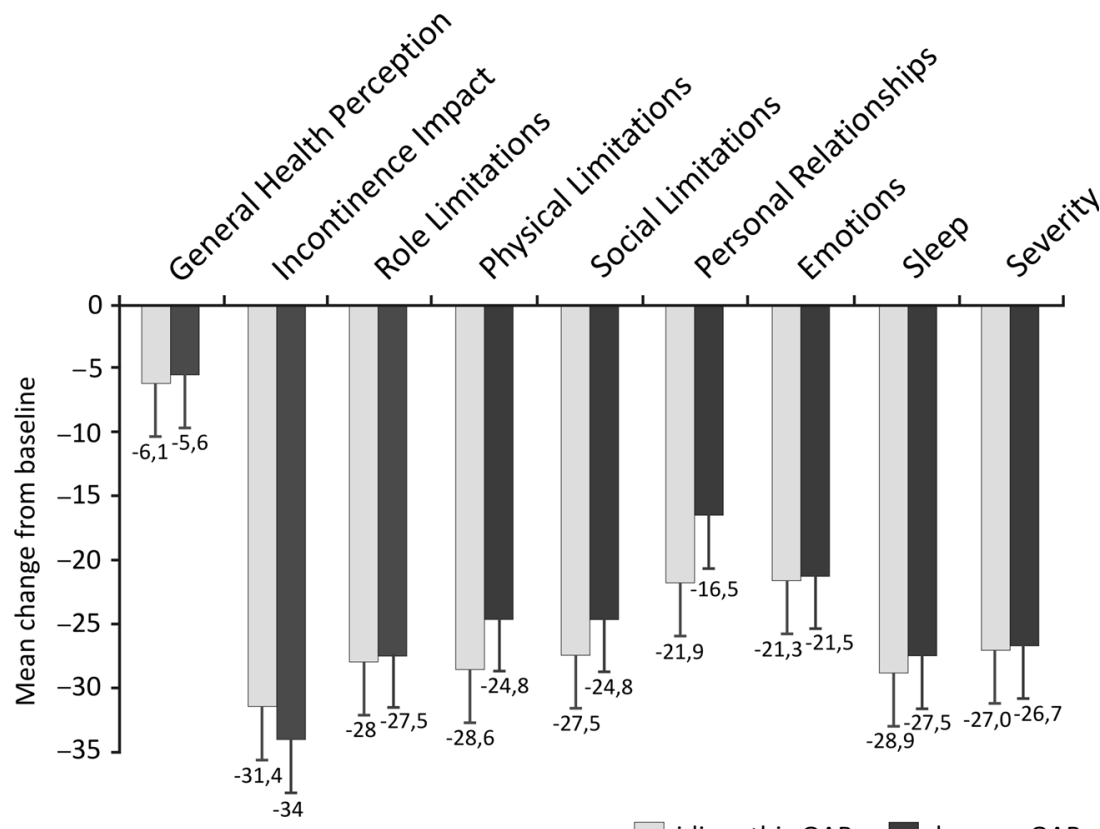

idiopathic $O A B$ de novo $O A B$ in all domains of the KHQ in both groups, and again our results are consistent with published observations from large RCTs investigating idiopathic OAB $[15,16]$.

We observed UTIs in $4 \%$ of patients only, a rate considerably lower than in previous clinical trials [15]. The incidence of elevated PVR volumes was similar to results of previous trials $[15,16]$. Therefore, the low incidence of UTI may be attributable to our routine use of antimicrobial prophylaxis before and after cystoscopy and use of prophylaxis for all patients with elevated PVR volumes.

We found only one previous comparative study assessing the efficacy of pharmacological therapy in women with de novo $(n=110)$ and idiopathic $(n=120)$ OAB [14]. Patients in both groups were treated with $5 \mathrm{mg}$ of solifenacin taken orally once daily for 3 months. In that prior study, patients in the de novo group reported significantly worse subjective and objective results after 3 months. More effective treatment options are needed for patients with de novo OAB symptoms, and overall, our results confirm the efficacy and safety of onabotulinumtoxinA in this setting.

The strengths of our study include the excellent follow -up achieved in a highly pragmatic and generalizable setting. Limitations include the relatively small number of patients and lack of placebo control. Lack of statistical significance for comparisons between groups must therefore be interpreted with caution considering the number of participants. Our findings may be subject to bias from unmeasured confounding, necessitating future randomized trials.

We observed that onabotulinumtoxin A injection was both effective and safe for patients with de novo OAB after MUS surgery, with comparable results to women with idiopathic OAB. Injections increased voided volume, decreased urinary frequency and number of UUI episodes, and improved QoL. Although the study provides limited power to test differences

Table 3 Incidence of adverse events at weeks 2 and 12 after onabotulinumtoxinA injections

\begin{tabular}{lllll}
\hline Adverse event & $\begin{array}{l}\text { Idiopathic OAB } \\
(n=53) \\
(\text { at week 2) }\end{array}$ & $\begin{array}{l}\text { Idiopathic OAB } \\
(n=53) \\
(\text { at week 12) }\end{array}$ & $\begin{array}{l}\text { De novo OAB after } \\
\text { MUS }(n=49) \\
\text { (at week 2) }\end{array}$ & $\begin{array}{l}\text { De novo OAB after } \\
\text { MUS }(n=49)(\text { at week 12) }\end{array}$ \\
\hline Urinary tract infection & $2(3.7)$ & $1(1.8)$ & $2(4.0)$ & $1(2.0)$ \\
Hematuria and/or leukocyturia & $1(1.8)$ & $0(0)$ & $3(6.1)$ & $1(2.0)$ \\
Weakness and fatigue & $0(0)$ & $0(0)$ & $1(2.0)$ & $0(0)$ \\
Residual urine volume $<100 \mathrm{ml}$ & $20(37.7)$ & $5(9.4)$ & $13(26.5)$ & $7(14.2)$ \\
Residual urine volume $>100 \mathrm{ml}$ and $<200 \mathrm{ml}$ & $7(13.2)$ & $2(3.7)$ & $12(24.5)$ & $3(6.1)$ \\
Residual urine volume $>200 \mathrm{ml}$ and $<350 \mathrm{ml}$ & $1(1.9)$ & $0(0)$ & $1(2.0)$ & $0(0)$ \\
Urine retention $>350 \mathrm{ml}$ & $0(0)$ & $0(0)$ & $2(4.0)$ & $0(0)$
\end{tabular}

Differences between investigated groups were not statistically significant. Data presented as $n(\%)$

$O A B$ overactive bladder, MUS midurethral sling 
in adverse events between groups, the rates of retention and requirement for catheterization even among women with a prior midurethral sling were acceptable. Patients with de novo $\mathrm{OAB}$ must be warned about the potential increased risk of urinary retention. These observational data provide evidence that onabotulinumtoxinA injections can effectively treat patients with de novo $\mathrm{OAB}$ following incontinence surgery.

Acknowledgments This research was an observational study and was not supported by any pharmaceutical company.

Conflicts of interest P. Miotla: speaker, paid investigator for Allergan, Astellas, Bayer

K. Futyma: speaker, paid investigator for Allergan, Astellas, Bayer

R. Cartwright: no disclosures

M. Bogusiewicz: paid investigator for Astellas

K. Skorupska: paid investigator for Astellas, Bayer

E. Markut-Miotla: no disclosures

T. Rechberger: speaker, paid investigator for Allergan, Astellas, Bayer

Open Access This article is distributed under the terms of the Creative Commons Attribution 4.0 International License (http://creativecommons.org/licenses/by/4.0/), which permits unrestricted use, distribution, and reproduction in any medium, provided you give appropriate credit to the original author(s) and the source, provide a link to the Creative Commons license, and indicate if changes were made.

\section{References}

1. Raman SV, Raker CA, Sung VW (2014) Concomitant apical prolapse repair and incontinence procedures: trends from 2001-2009 in the United States. Am J Obstet Gynecol 211:222.e1-222.e5

2. Wu JM, Matthews CA, Conover MM, Pate V, Jonsson Funk M (2014) Lifetime risk of stress urinary incontinence or pelvic organ prolapse surgery. Obstet Gynecol 123:1201-1206

3. Palva K, Nilsson CG (2011) Prevalence of urinary urgency symptoms decreases by mid-urethral sling procedures for treatment of stress incontinence. Int Urogynecol J22:1241-1247

4. Petri E, Ashok K (2012) Complications of synthetic slings used in female stress urinary incontinence and applicability of the new IUGA-ICS classification. Eur J Obstet Gynecol Reprod Biol 165: 347-351

5. Serati M, Ghezzi F, Cattoni E et al (2012) Tension-free vaginal tape for the treatment of urodynamic stress incontinence: efficacy and adverse effects at 10-year follow-up. Eur Urol 61:939-946

6. Lee JK, Dwyer PL, Rosamilia A, Lim YN, Polyakov A, Stav K (2013) Which women develop urgency or urgency urinary incontinence following midurethral slings? Int Urogynecol J 24:47-54
7. Bogusiewicz M, Monist M, Gałczyński K, Woźniak M, Wieczorek AP, Rechberger T (2014) Both the middle and distal sections of the urethra may be regarded as optimal targets for 'outside-in' transobturator tape placement. World J Urol 32:1605-1611

8. Wang F, Song Y, Huang H (2009) Which placement of the tensionfree vaginal tape is more important for urinary continence: midurethral position or bladder neck? Consideration from a case report. Int Urogynecol J Pelvic Floor Dysfunct 20:1277-1279

9. Bogusiewicz M, Monist M, Stankiewicz A, Woźniak M, Wieczorek AP, Rechberger T (2013) Most of the patients with suburethral sling failure have tapes located outside the high-pressure zone of the urethra. Ginekol Pol 84:334-338

10. Stewart WF, Corey R, Herzog AR et al (2001) Prevalence of overactive bladder in women: results from the NOBLE program. Int Urogynecol J 12:66

11. Agarwal A, Eryuzlu LN, Cartwright R et al (2014) What is the most bothersome lower urinary tract symptom? Individual- and population-level perspectives for both men and women. Eur Urol 65:1211-1217

12. Robinson D, Giarenis I, Cardozo L (2013) The management of overactive bladder refractory to medical therapy. Maturitas 75 : 101-104

13. Rechberger T, Kulik-Rechberger B, Miotła P, Wrobel A (2014) The new era in the pharmacological treatment of overactive bladder (OAB): mirabegron-a new selective beta3agonist. Ginekol Pol 853:214-219

14. Serati M, Braga A, Sorice P, Siesto G, Salvatore S, Ghezzi F (2014) Solifenacin in women with de novo overactive bladder after tension-free obturator vaginal tape-is it effective? J Urol 191: 1322-1326

15. Chapple C, Sievert KD, MacDiarmid S et al (2013) OnabotulinumtoxinA $100 \mathrm{U}$ significantly improves all idiopathic overactive bladder symptoms and quality of life in patients with overactive bladder and urinary incontinence: a randomised, double-blind, placebo-controlled trial. Eur Urol 64:249-256

16. Fowler CJ, Auerbach S, Ginsberg D et al (2012) OnabotulinumtoxinA improves health-related quality of life in patients with urinary incontinence due to idiopathic overactive bladder: a 36-week, double-blind, placebo-controlled, randomized, dose-ranging trial. Eur Urol 62:148-157

17. Sievert KD, Chapple C, Herschorn S et al (2014) OnabotulinumtoxinA $100 \mathrm{U}$ provides significant improvements in overactive bladder symptoms in patients with urinary incontinence regardless of the number of anticholinergic therapies used or reason for inadequate management of overactive bladder. Int $\mathrm{J}$ Clin Pract 68:1246-1256

18. Nitti VW, Dmochowski R, Herschorn S, EMBARK Study Group et al (2013) OnabotulinumtoxinA for the treatment of patients with overactive bladder and urinary incontinence: results of a phase 3 , randomized, placebo controlled trial. J Urol 189:2186-2193 